\title{
Umbilical cord arterial blood gas analysis in term singleton pregnancies: a retrospective analysis over 11 years
}

\author{
Ji Hee Lee, MD, Jihee Jung, MD, Hyea Park, MD, Seo-yeon Kim, MD, Do youn Kwon, MD, \\ Suk-Joo Choi, MD, PhD, Soo-young Oh, MD, PhD, Cheong-Rae Roh, MD, PhD \\ Department of Obstetrics and Gynecology, Samsung Medical Center, Sungkyunkwan University School of Medicine, Seoul, Korea
}

\section{Objective}

Given that the large volume of data on cord arterial blood gas analysis (ABGA) have been rarely addressed in Korean population, we aimed to examine the incidence, associated factors, and neonatal outcomes in cases of low cord pH, and investigate the incidence of cerebral palsy (CP).

\section{Methods}

From data of all consecutive term singleton pregnancies delivered in our institution from 2006 to 2016 ( $n=15,701)$, cases with cord ABGA $(n=14,221)$ available were included. We collected information on maternal clinical characteristics and delivery outcomes and also examined neonatal and infant outcomes, including neonatal intensive care unit (NICU) admission and $\mathrm{CP}$, in cases with low cord $\mathrm{pH}$, defined as a $\mathrm{pH}<7.1$.

\section{Results}

Rates of low Apgar scores at 1 minute $(<4)$ and 5 minutes $(<7)$ were $0.6 \%(n=79)$ and $0.4 \%(n=58)$, respectively. Rates of cord pH $<7.2,<7.1$, and $<7.0$ were $7.1 \%(n=1,011), 1.1 \%(n=163)$, and $0.3 \%(n=38)$, respectively. Among cases with low cord $\mathrm{pH}, 30.1 \%(\mathrm{n}=49 / 163)$ were admitted to the NICU and $11.0 \%(n=18 / 163)$ required ventilator support. Ultrasonography of the brain was performed in $28.8 \%(n=47 / 163)$, with abnormal findings observed in $27.7 \%$ $(n=13 / 47)$. Among cases with low cord $\mathrm{pH}, 1.8 \%(n=3 / 163)$ were subsequently diagnosed with $\mathrm{CP}$, including 2 cases of spastic CP and 1 of ataxic CP.

\section{Conclusion}

Although low cord $\mathrm{pH}$ was a relatively frequent finding observed in 1 out of every 87 cases, hypoxic-ischemic encephalopathy-related CP was found in only 1 out of 7,111 term singleton deliveries over 11 years in our institution.

Keywords: Umbilical cord; Analysis, blood gas; Term birth; Cerebral palsy

\section{Introduction}

While most fetuses experience the physiologic fetal-to-neonatal transition to air breathing after birth at term, $10 \%$ of newborns require some degree of active resuscitation to activate breathing and $1 \%$ require extensive care [1]. According to the most recent neonatal resuscitation protocol endorsed by the American Academy of Pediatrics (AAP) and supported by published studies, it was reported that approximately $3 \%$ of newborns needed positive-pressure ventilation, $2 \%$ needed endotracheal intubation, and $0.1 \%$ needed cardiac
Received: 2019.09.19. Revised: 2019.11.12. Accepted: 2019.11.17. Corresponding author: Soo-young $\mathrm{Oh}, \mathrm{MD}, \mathrm{PhD}$

Department of Obstetrics and Gynecology, Samsung Medical Center, Sungkyunkwan University School of Medicine, 81 Ilwonro, Gangnam-gu, Seoul 06351, Korea

E-mail: ohsymd@skku.edu https://orcid.org/0000-0003-3002-0048

The abstract of this study was presented as an oral presentation at $103^{\text {rd }}$ Annual Meeting of the Korean Society of Obstetrics and Gynecology (22 September 2017).

Articles published in Obstet Gynecol Sci are open-access, distributed under the terms of the Creative Commons Attribution Non-Commercial License (http://creativecommons. org/licenses/by-nc/3.0/) which permits unrestricted non-commercial use, distribution, and reproduction in any medium, provided the original work is properly cited.

Copyright $\odot 2020$ Korean Society of Obstetrics and Gynecology 


\title{
Obstetrics \& Gynecology Science
}

\author{
Vol. 63, No. 3, 2020
}

compressions or epinephrine administration [2-4].

Difficulty in initiating respiration, and depression of tone and reflexes are common symptoms of neonatal encephalopathy (NE), which is a clinically defined syndrome of disturbed neurologic function in the earliest days of life in babies born at or beyond 35 weeks of gestation manifested by a subnormal level of consciousness or seizures. Historically, the incorrect assumption that most NE results from hypoxia during the intrapartum period has hindered serious research into other possible causes of NE. Recent epidemiologic studies have identified several preconception demographic, and maternal medical conditions (advanced maternal age, family history of seizure, family history of neurologic disorder, maternal thyroid disease, and infertility treatment) and antepartum risk factors (preeclampsia, moderate to severe vaginal bleeding, advancing pregnancy beyond 39 weeks gestation, late or no prenatal care, and low neonatal birth weight percentile) as independent risk factors for NE [5-7]. Of note, it was reported that $70 \%$ of NE cases were likely the result of events arising before the onset of labor [8].

Hypoxic ischemic encephalopathy (HIE) comprises a causespecific subset of all NE. Inaccurate prediction of fetal acidemia by intrapartum fetal heart rate (FHR) monitoring can lead to the presumptive diagnosis of HIE as the direct cause of depressed neonates in cases that are otherwise unexplained, thereby overestimating the true incidence of HIE. According to the American College of Obstetricians and Gynecologists (ACOG) and the AAP, a low cord arterial blood pH $<7.0$ and/ or a base excess $(\mathrm{BE})<-12 \mathrm{mmol} / \mathrm{L}$ are the generally accepted cutoff values for pathological acidosis, which increases the risk of seizures, HIE, and cerebral palsy (CP) [9]. As umbilical cord blood gas and acid-base assessment are the most objective predictors of fetal acidemia $[10,11]$, it is usually recommended by the ACOG to obtain a cord arterial blood gas analysis (ABGA) in certain clinical circumstances such as a low 5-minute Apgar score, severe growth restriction, or abnormal FHR tracing $[12,13]$, although there is no specific guideline in Korea.

Meanwhile, as a tertiary center, our institution has adopted the routine performance of cord blood ABGA at birth for every delivery since 2006 for a variety of reasons, including medicolegal considerations. Surprisingly, we could not find any study with a large volume of data including Korean population regarding cord $A B G A$ at birth and the association analysis between cord $\mathrm{pH}$ and other clinical factors such as meconium staining, nuchal cord, and mode of delivery. This led us to initiate a comprehensive analysis of cord blood $\mathrm{pH}$ in consecutive singleton pregnancies delivered at term in our hospital population.

The specific questions that we pursued here were as follows. First, what are the distribution of cord blood $\mathrm{pH}$ and the incidence of low cord $\mathrm{pH}$ ? Second, what are the associations between low cord blood $\mathrm{pH}$ and clinical factors including the grade of meconium, number of nuchal cord cases, and mode of delivery? Thirdly, among cases with low cord $\mathrm{pH}$ defined as a $\mathrm{pH}<7.1$, what is the actual frequency of adverse neonatal outcomes including neonatal intensive care unit (NICU) admission, ventilator support, need for brain ultrasonography, and abnormal sonographic findings? Lastly, how many CP cases were subsequently diagnosed among neonates with low cord $\mathrm{pH}$ at term?

\section{Materials and methods}

During the study period from January 2006 to December 2016, there were 21,428 deliveries in our institution including 4,855 preterm deliveries, 16,554 term deliveries, and 19 post-term deliveries. Fig. 1 shows the inclusion and exclu-

Total 21,428 deliveries

(Study period: Jan 2006 Dec 2016)

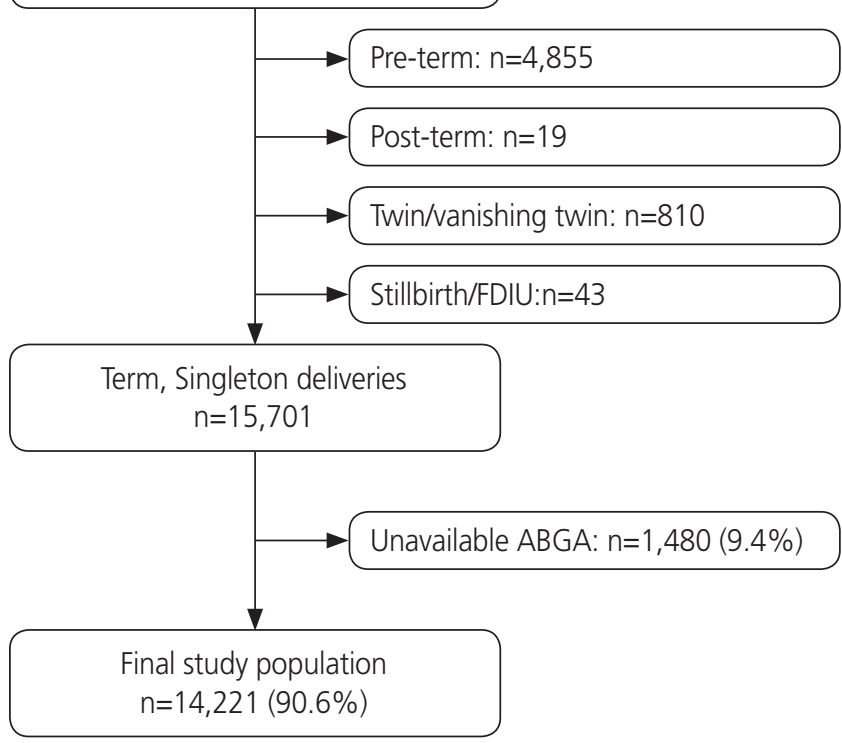

Fig. 1. Study population with inclusion and exclusion criteria. FDIU, Fetal death in utero; ABGA, arterial blood gas analysis; VBGA, venous blood gas analysis. 


\section{Obstetrics \& Gynecology Science}

Ji Hee Lee, et al. Cord arterial blood gas analysis in term pregnancies

Table 1. Comparison of clinical characteristics according to cord blood $\mathrm{pH}$ at birth

\begin{tabular}{|c|c|c|c|c|}
\hline Clinical variables & $\begin{array}{c}\text { Total } \\
(n=14,221)\end{array}$ & $\begin{array}{c}\text { Cord } \mathrm{pH} \geq 7.1 \\
(n=14,058)\end{array}$ & $\begin{array}{c}\text { Cord } p H<7.1 \\
(n=163)\end{array}$ & $P$-value \\
\hline Maternal age (yr) & $32.4 \pm 3.8$ & $32.3 \pm 3.9$ & $32.4 \pm 3.8$ & $0.813^{\mathrm{al}}$ \\
\hline Maternal height $(\mathrm{cm})^{\mathrm{b})}$ & $161.6 \pm 5.3$ & $161.6 \pm 5.3$ & $160.6 \pm 5.3$ & $0.024^{a)}$ \\
\hline Maternal weight $(\mathrm{kg})^{\mathrm{b})}$ & $68.2 \pm 9.5$ & $68.2 \pm 9.5$ & $65.9 \pm 9.4$ & $0.006^{a)}$ \\
\hline Maternal BMI $\left(\mathrm{kg} / \mathrm{m}^{2}\right)^{\mathrm{b})}$ & $26.1 \pm 3.3$ & $26.1 \pm 3.3$ & $25.5 \pm 3.5$ & $0.057^{\mathrm{a})}$ \\
\hline Primiparity & $7,597(53.4)$ & $7,484(53.2)$ & $113(69.3)$ & $<0.001^{\mathrm{c})}$ \\
\hline Prior preterm delivery & $684(4.8)$ & $679(4.8)$ & $5(3.1)$ & $0.199^{c}$ \\
\hline Underlying hypertension & $185(1.3)$ & $183(1.3)$ & $2(1.2)$ & $1.000^{c)}$ \\
\hline Hypertensive disorders ${ }^{\mathrm{d})}$ & $482(3.4)$ & $473(3.4)$ & $9(5.5)$ & $0.130^{c}$ \\
\hline Overt DM & $100(0.7)$ & $99(0.7)$ & $1(0.6)$ & $1.000^{c)}$ \\
\hline Gestational DM & $926(6.5)$ & $915(6.5)$ & $11(6.7)$ & $1.000^{c)}$ \\
\hline GA at delivery (wk) & & & & $0.369^{e)}$ \\
\hline $37.0-37.6$ & $1,415(10.0)$ & $1,394(9.9)$ & $21(12.9)$ & \\
\hline $38.0-38.6$ & $4,075(28.7)$ & $4,042(28.8)$ & $33(20.2)$ & \\
\hline $39.0-39.6$ & $4,341(30.5)$ & $4,292(30.5)$ & $49(30.1)$ & \\
\hline $40.0-40.6$ & $3,378(23.7)$ & $3,335(23.7)$ & $43(26.4)$ & \\
\hline $41.0-41.6$ & $1,012(7.1)$ & $995(7.1)$ & $17(10.4)$ & \\
\hline Meconium staining grade & & & & $0.001^{\mathrm{e})}$ \\
\hline None & $12,769(89.8)$ & $12,660(90.0)$ & $109(66.9)$ & \\
\hline+ & $1,080(7.6)$ & $1,049(7.5)$ & $31(19.0)$ & \\
\hline++ & $223(1.6)$ & $213(1.5)$ & $10(6.1)$ & \\
\hline+++ & $113(0.8)$ & $106(0.8)$ & $7(4.3)$ & \\
\hline++++ & $36(0.2)$ & $30(0.2)$ & $6(3.7)$ & \\
\hline Number of nuchal cord & & & & $0.002^{e)}$ \\
\hline 0 & $9,894(69.6)$ & $9,801(69.7)$ & $93(57.1)$ & \\
\hline 1 & $3,697(26.0)$ & $3,636(25.9)$ & $61(37.4)$ & \\
\hline 2 & $546(3.8)$ & $538(3.8)$ & $8(4.9)$ & \\
\hline 3 & $73(0.5)$ & $73(0.5)$ & $0(0.0)$ & \\
\hline 4 & $11(0.1)$ & $10(0.1)$ & $1(0.6)$ & \\
\hline \multicolumn{5}{|l|}{ Mode of delivery } \\
\hline Elective CS & 2,925 (20.6) & $2,914(20.7)$ & $11(6.7)$ & $<0.001^{c)}$ \\
\hline Emergent CS & $1,809(12.7)$ & $1,779(12.6)$ & $30(18.4)$ & $0.028^{c)}$ \\
\hline Spontaneous VD & $8,712(61.3)$ & $8,612(61.3)$ & $100(61.4)$ & $0.981^{c)}$ \\
\hline Vacuum-assisted VD & $775(5.4)$ & $753(5.4)$ & $22(13.5)$ & $<0.001^{\mathrm{c})}$ \\
\hline Male sex of baby & $7,276(51.2)$ & $7,185(51.1)$ & $91(55.8)$ & $0.231^{c}$ \\
\hline Apgar score at $1 \mathrm{~min}<4$ & $79(0.6)$ & $53(0.4)$ & $26(16.0)$ & $<0.001^{\mathrm{c})}$ \\
\hline Apgar score at 5 min $<7$ & $58(0.4)$ & $39(0.3)$ & $19(11.7)$ & $<0.001^{\mathrm{c})}$ \\
\hline Neonatal weight (kg) & $3.24(1.51-5.19)$ & $3.24(1.51-5.19)$ & $3.08(1.68-4.62)$ & $<0.001^{f)}$ \\
\hline SGA & $1,559(11.0)$ & $1,523(10.8)$ & $36(22.1)$ & $<0.001^{\mathrm{c})}$ \\
\hline LGA & $1,226(8.6)$ & $1,218(8.7)$ & $8(4.9)$ & $0.089^{c)}$ \\
\hline NICU admission & $851(6.0)$ & $802(5.7)$ & $49(30.1)$ & $<0.001^{\mathrm{c})}$ \\
\hline
\end{tabular}

Data are presented as the mean \pm standard deviation or number (\%) or median (range).

BMI, body mass index; DM, diabetes mellitus; GA, gestational age; CS, caesarean section; VD, vaginal delivery; SGA, small for gestational age; LGA, large for gestational age; NICU, neonatal intensive care unit.

${ }^{\text {a) }} P$-value by Student's $t$-test; ${ }^{b}$ Cases unavailable with maternal height $(n=3,149)$, weight $(n=2,939)$, and BMI $(n=3,242)$ were excluded from this analysis; ${ }^{c}$ P-value by chi-squared test; ${ }^{d)}$ Hypertensive disorders include gestational hypertension, mild pre-eclampsia, severe pre-eclampsia, superimposed pre-eclampsia on chronic hypertension, HELLP syndrome (hemolysis, elevated liver enzymes, and a low platelet count), and eclampsia; ${ }^{e} P$-value by linear-by-linear association; ${ }^{\dagger} P$-value by Mann-Whitney $U$ test; $P<0.05$ : statistically significant. 


\section{Obstetrics \& Gynecology Science}

Vol. 63, No. 3, 2020

sion protocols of this study population. We excluded cases with preterm birth, post-term birth, twin pregnancy including vanishing twin, stillbirth and fetal death in utero. After the exclusions, there were 15,701 term singleton pregnancies. Among them, the results of umbilical cord ABGA were available in 14,221 cases $(90.6 \%)$, which constituted the final population of this study. We collected data on clinical variables including maternal age, height, weight, parity, history of prior preterm delivery, gestational age at delivery, the presence of meconium staining, the number of nuchal cord, mode of delivery, sex of baby, cord blood pH, Apgar scores at 1 minute and 5 minutes, neonatal birth weight, and NICU admission. Data were acquired from the electronic medical record system in our institution. The grade of meconium staining was divided into the following 5 groups by experienced labor and delivery nurses: none, $1+, 2+, 3+$, and $4+$; nuchal cord was graded on a scale of 0 to 4 . Mode of delivery was divided into 4 groups: elective caesarean section delivery, emergent caesarean section delivery, spontaneous vaginal delivery, and vacuum-assisted vaginal delivery. Apgar scores were assigned by experienced labor and delivery nurses or pediatric doctors. The cord blood was sampled immediately after delivery with heparinized syringes and analyzed within 30 minutes in most cases. We defined low cord pH as a $\mathrm{pH}<7.1$. Neonatal weight was categorized into 3 groups: small for gestational age (SGA), appropriate for gestational age (AGA), and large for gestational age (LGA) according to national data from the Korean Health Insurance Review and Assessment Service 2009.

In cases of low cord pH, we also examined additional neonatal and infant outcomes including ventilator support, brain ultrasonographic findings, major anomaly, and CP by thorough review of medical records. The major anomaly category included heart anomaly, central nervous system anomaly, chromosomal anomaly, specific syndromes, and complicated cleft palate. CP was identified based on the diagnosis by the pediatric or rehabilitation departments during the infant's follow-up period. We also searched for all cases of neonates born with normal cord $\mathrm{pH}(\geq 7.1)$ during the same study period who were diagnosed with CP during their follow-up period by screening the International Classification of Diseases, Tenth Revision, Clinical Modification (ICD-10-CM) infant codes (G80; CP) in our electronic medical system. Case confirmation was based on the clinical diagnosis made by pediatric or rehabilitation doctors as documented in the medical

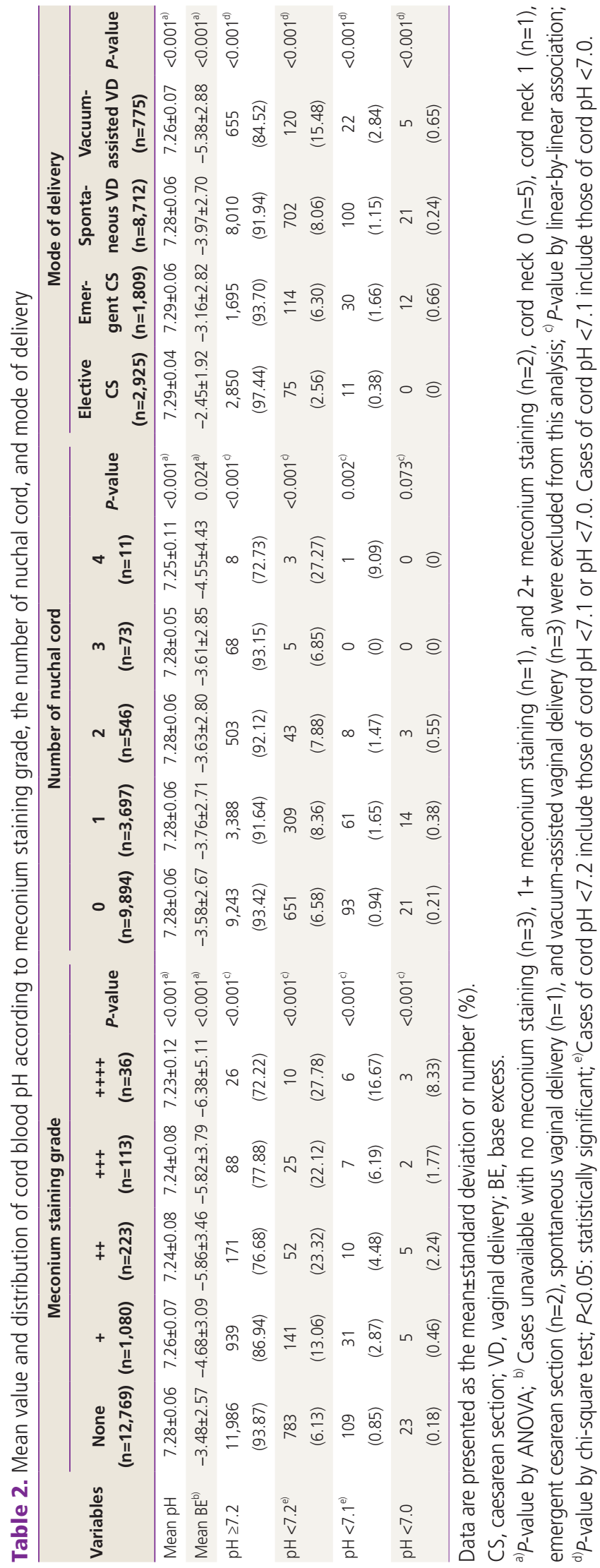




\section{Obstetrics \& Gynecology Science}

Ji Hee Lee, et al. Cord arterial blood gas analysis in term pregnancies

record. Statistical analysis was done using Mann-Whitney $U$ tests, analysis of variance tests, Student's $t$-tests, and linearby-linear association tests using SPSS software (version 25.0; IBM Corp., Armonk, NY, USA).

\section{Results}

Table 1 shows a comparison of the clinical characteristics according to cord blood $\mathrm{pH}$ at birth. The low cord $\mathrm{pH}$ group was characterized by lower maternal height and weight and a higher rate of primiparity. The low cord $\mathrm{pH}$ group was associated with a higher grade of meconium staining and higher number of nuchal cord compared to the control group. In terms of the mode of delivery, the low cord pH group showed a significantly lower rate of elective caesarean section and higher rates of emergent caesarean section and vacuum-assisted delivery compared to the control group. Overall, rates of low Apgar scores at 1 minute $(<4)$ and 5 minutes $(<7)$ were $0.6 \%(n=79)$ and $0.4 \%(n=58)$, respectively. The low cord $\mathrm{pH}$ group was also associated with a higher rate of low Apgar scores at 1 minute and 5 minutes and NICU admission compared to the control group. Of note, the low cord pH group showed a lower mean neonatal weight $(3.08$ [1.68, 4.62] kg vs. 3.24 [1.51, 5.19] kg; $P<0.001)$ and a higher rate of SGA compared to the control group $(22.1 \%$ vs. $10.8 \% ; P<0.001)$.

In our study population of term singleton pregnancies, the mean cord blood pH was $7.28 \pm 0.06$, ranged $6.75-7.53$ and the mean BE was $-3.63 \pm 2.69$. Rates of cord $\mathrm{pH}<7.2,<7.1$, and $<7.0$ were $7.1 \%(n=1,011), 1.1 \%(n=163)$, and $0.3 \%$ $(n=38)$, respectively. Next, we analyzed the mean value and distribution of cord blood $\mathrm{pH}$ according to the grade of meconium staining, number of nuchal cord, and mode of delivery (Table 2). There were significant differences in the mean cord $\mathrm{pH}$ based on the grade of meconium staining, number of nuchal cord, and mode of delivery ( $P<0.001$ for all), but all mean values were within the normal range. Severe grades of meconium staining were associated with higher rates of cord $\mathrm{pH}<7.2,<7.1$, and $<7.0$. The incidence of nuchal cord was also associated with a higher rate of cord $\mathrm{pH}<7.2$ and $<7.1$ but not with cord $\mathrm{pH}<7.0$. There were also significant differences in the rates of cord $\mathrm{pH}<7.2,<7.1$, and $<7.0$ according to the mode of delivery.

Fig. 2 shows the clinical course information in cases with low cord blood pH. Among 163 cases, 49 babies (30.1\%)

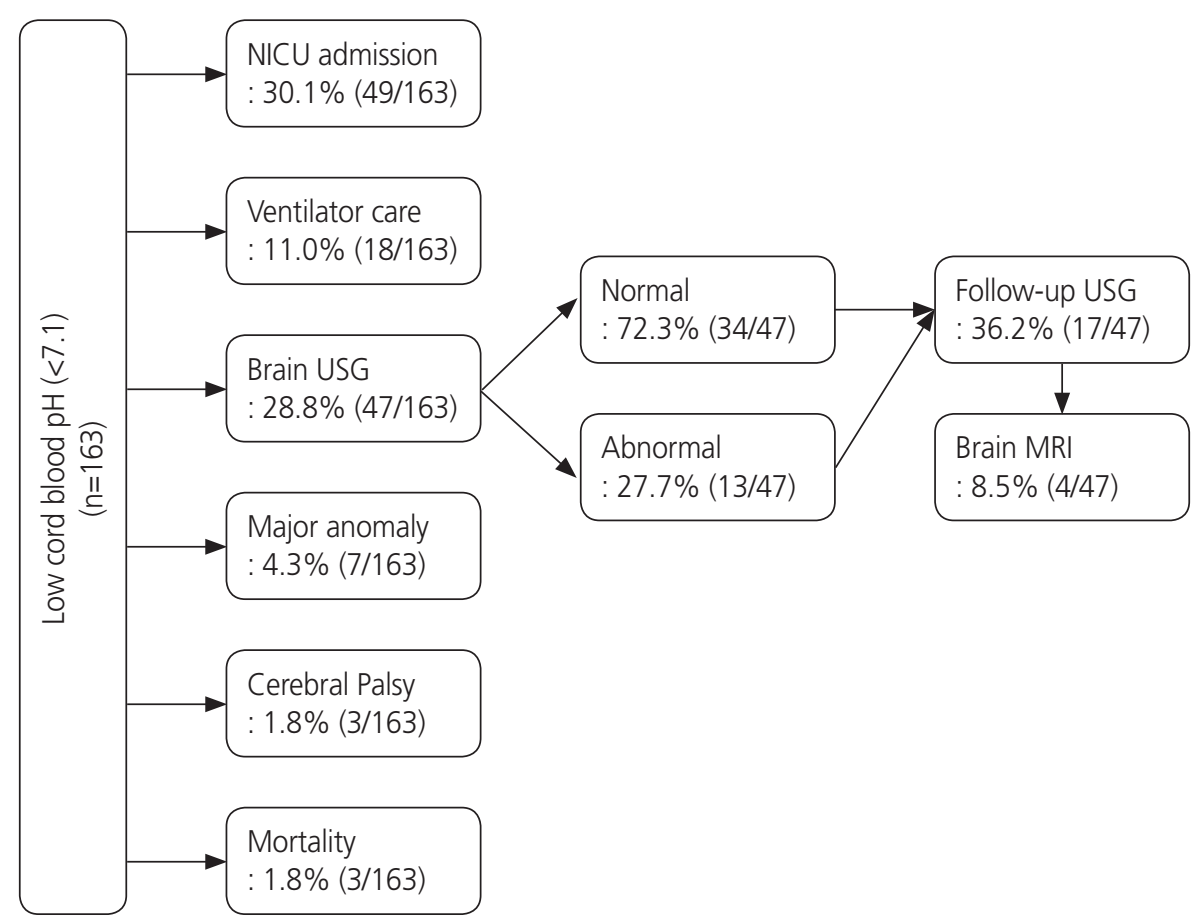

Fig. 2. Clinical course and information in cases with low cord blood pH. NICU, neonatal intensive care unit; USG, ultrasonography; MRI, magnetic resonance imaging. 


\section{Obstetrics \& Gynecology Science}

Vol. 63, No. 3, 2020

were admitted to the NICU and 18 (11.0\%) needed ventilator support. Ultrasonography of the brain was performed in $28.8 \%(n=47)$ of cases, and significant abnormal findings were observed in $27.7 \%(n=13)$ as follows: diffuse brain edema $(n=7)$, grade 2 intraventricular hemorrhage $(n=2)$, basal ganglia vasculopathy $(n=2)$, Dandy-Walker complex $(n=1)$, and myelination disorder $(n=1)$.

Table 3 presents detailed clinical information on 3 cases of presumed $\mathrm{CP}$ with low cord $\mathrm{pH}$. The baby in the first case was born at 39.0 weeks by prompt emergent caesarean section due to a non-reassuring FHR pattern detected at the time of admission. The neonatal weight was $3.234 \mathrm{~kg}$ and the meconium staining grade was 4+. The Apgar scores at 1 minute and 5 minutes were 0 and 4, respectively, but increased to 7 at 10 minutes. Cord blood pH was 7.021, and BE was $-15.00 \mathrm{mmol} / \mathrm{L}$. The baby was admitted into the NICU immediately and placed on a ventilator. An ultrasound examination of the brain on the first day of life revealed profound HIE findings, and the baby was diagnosed with moderate-severe cerebral coordination disturbance 1 month later. The baby was discharged 23 days after birth and, unfortunately, was found dead at home 6 months later. The second case was born by emergent caesarean section due to a non-reassuring FHR pattern developed during the second stage. The neonatal weight was $3.392 \mathrm{~kg}$, and meconium staining grade was $1+$. The Apgar scores at 1 minute and 5 minutes were 2 and 5, respectively. Cord blood pH was 6.887, and the BE was $-18.30 \mathrm{mmol} / \mathrm{L}$. The baby was admitted to the NICU and placed on a ventilator. Magnetic resonance imaging (MRI) of the brain checked on the 9th day showed a selective deep gray or white matter injury probably due to profound asphyxia. The electroencephalogram finding was also suggestive of diffuse cerebral dysfunction. The baby was lost to follow-up but was presumed to have CP. The third baby was born by spontaneous vaginal delivery without any remarkable event in the perinatal period. The baby had routine nursery care and was discharged uneventfully. However, after 4 months, the baby visited the pediatric clinic in our hospital due to difficulty with head tilting. Unfortunately, the baby suffered severe developmental delays and was finally diagnosed with ataxic CP at age 2.5 years, which was not HIE-related CP.

Lastly, we summarized the perinatal characteristics of the $\mathrm{CP}$ cases $(\mathrm{n}=18)$ with normal cord $\mathrm{pH}(\geq 7.1)$ at birth during

Table 3. $\mathrm{CP}$ cases among low cord pH at birth

\begin{tabular}{lccc}
\hline Clinical variables & Case $\mathbf{1}$ & Case 2 & Case 3 \\
\hline Birth year & 2006 & 2007 & 2014 \\
Maternal age (yr) & 26 & 29 & 33 \\
GA at delivery (wk) & 39.0 & 41.4 & 40.0 \\
Mode of delivery & Emergent CS & Emergent CS & Spontaneous VD \\
Weight of baby (kg) & $3.234(\mathrm{AGA})^{\text {a) }}$ & $3.392(\mathrm{AGA})^{\text {a) }}$ & $3.280(\mathrm{AGA})^{\mathrm{a}}$ \\
Meconium staining grade & $4+$ & $1+$ & None \\
Cord blood pH & 7.021 & 6.887 & 6.969 \\
Cord blood base excess (mmo//L) & -15.00 & -18.30 & -7.30 \\
Apgar score at 1 min & 0 & 2 & 7 \\
Apgar score at 5 min & 4 & 5 & 9 \\
Apgar score at 10 min & 7 & NA & NA \\
NICU admission & Yes & Yes & No \\
Ventilator support & Yes & Yes & No \\
Brain USG) & Done & Done & NA \\
Diagnosis of CP & Yes & Presumed & Yes (Ataxic CP) \\
Mortality & Yes & No & No
\end{tabular}

$G A$, gestational age; $C S$, caesarean section; VD, vaginal delivery; AGA, appropriate for gestational age; NA, not assessed; NICU, neonatal intensive care unit; USG, ultrasonography; CP, cerebral palsy.

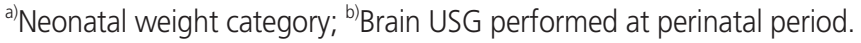


Obstetrics \& Gynecology Science

di Wee Lee, et al. Cord arterial blood gas analysis in term pregnancies

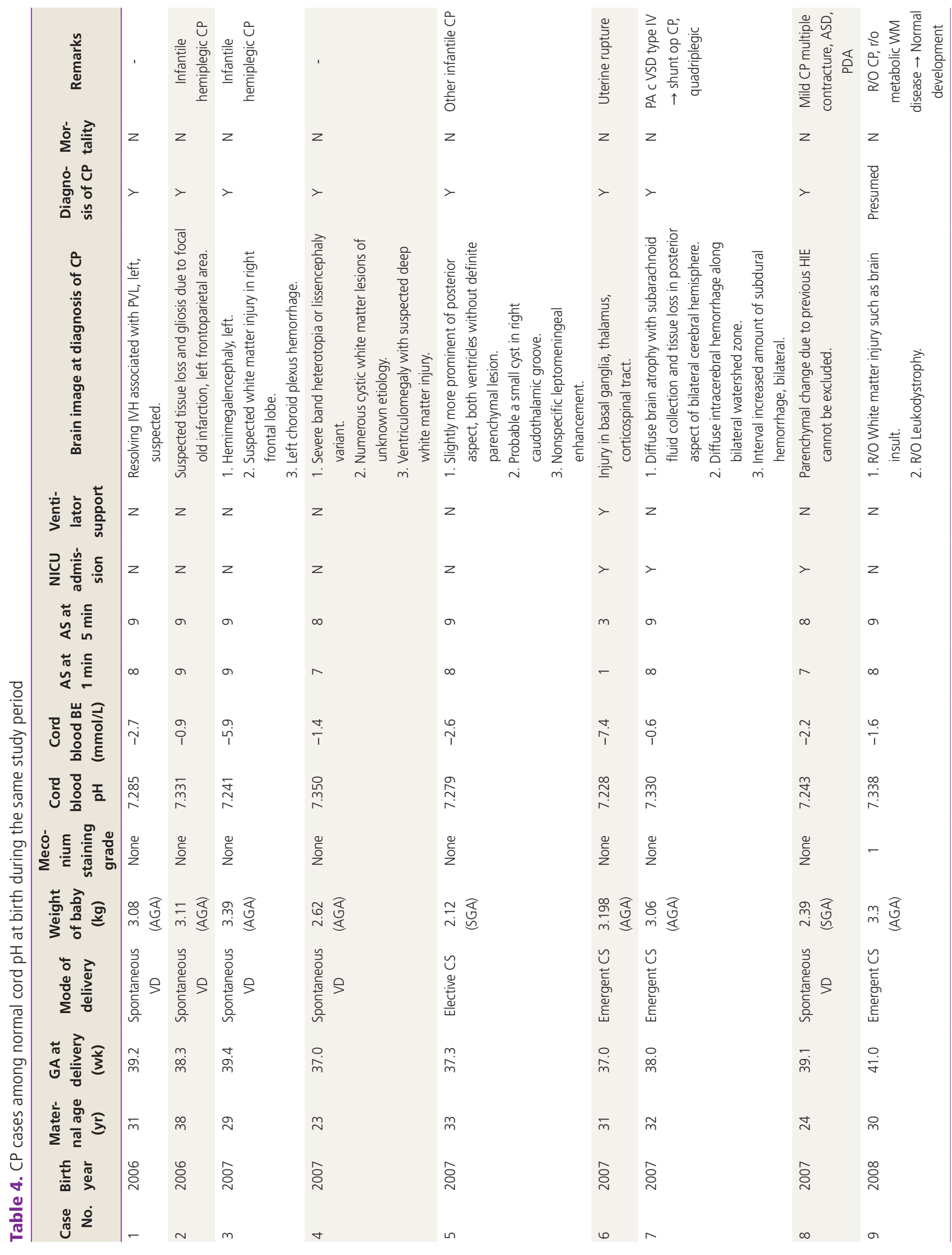

www.ogscience.org

299 


\section{Obstetrics \& Gynecology Science}

Vol. 63, No. 3, 2020

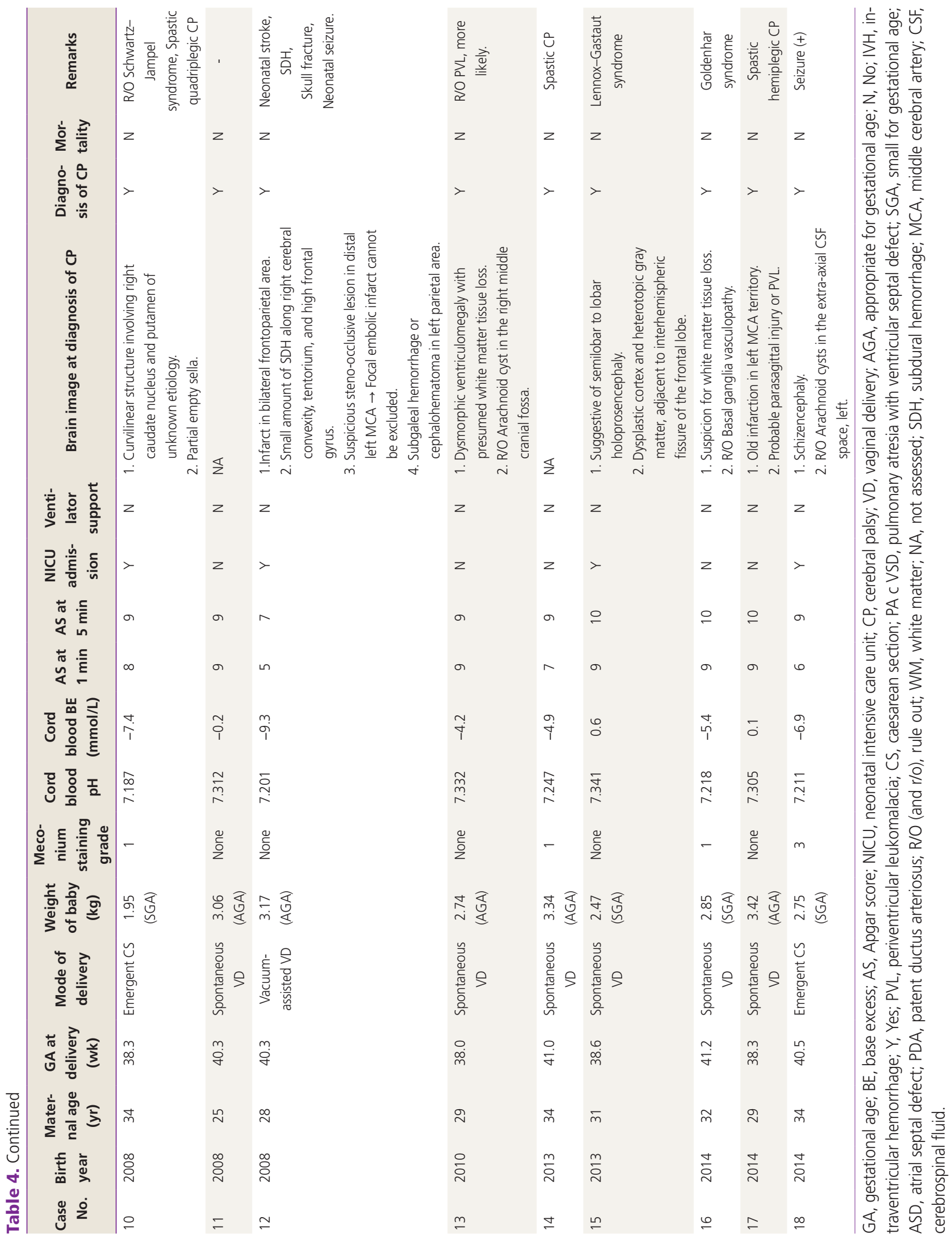




\section{Obstetrics \& Gynecology Science}

Ji Hee Lee, et al. Cord arterial blood gas analysis in term pregnancies

the same study period (Table 4). Among 18 cases of CP, there were 4 cases with brain anomalies (hemimegalencephaly, severe band heterotopia, semilobar to lobar holoprosencephaly, and schizencephaly), 3 cases with specific syndromes (Schwartz-Jampel syndrome, Lennox-Gastaut syndrome, and Goldenhar syndrome), 3 cases of old brain infarction, and 1 case with a cardiac anomaly. Overall, SGA was complicated in 6 cases. Of note, the cord $\mathrm{pH}$ and $\mathrm{BE}$ were all within normal ranges and only $38.9 \%(n=7 / 18)$ of cases had NICU admission during the neonatal period.

\section{Discussion}

Our data provided the following clinical information on a large hospital-based patient population over an 11-year study period. First, the mean cord blood pH was $7.28 \pm 0.06$ among term singleton pregnancies. The rates of cord $\mathrm{pH}<7.2,<7.1$, and $<7.0$ were $7.1 \%, 1.1 \%$, and $0.3 \%$, respectively. Secondly, low cord pH $(<7.1)$ was associated with multiple antepartum factors including lower maternal height and weight, a higher rate of primiparity, lower neonatal body weight, and a higher rate of SGA. As for intrapartum factors, the grade of meconium staining, number of nuchal cord, and mode of delivery were associated with low cord $\mathrm{pH}$, though the actual positive rate for low cord $\mathrm{pH}$ in the presence of these risk factors was quite low. Thirdly, among cases with low cord pH (<7.1), 30.1\% were admitted into the NICU, 11.0\% required ventilator support, and $28.8 \%$ warranted brain ultrasonography, with significant abnormal findings observed in $27.7 \%$ of cases. Lastly, the rate of presumable CP among cases with low cord pH was approximated at 1.8\% (3/163), with 2 cases of spastic CP and 1 case of ataxic CP.

Although it is well documented that an umbilical artery $\mathrm{pH}$ $<7.0$ is considered one of the prerequisite criteria for a subsequent diagnosis of HIE [14], some studies on cord pH and FHR monitoring have chosen to use an umbilical artery $\mathrm{pH}$ of $<7.1$ as the critical acid-base level in an effort to identify the predictive risk factors at the stage before injury occurs [15-17]. Similarly, as we aimed to investigate the neonatal outcomes in cases near the critical pH level, we defined "low" cord pH as $<7.1$ for research purposes. In our study, the mean cord blood $\mathrm{pH}$ and $\mathrm{BE}$ were $7.28 \pm 0.06$ and $-3.63 \pm 2.69 \mathrm{mmol} / \mathrm{L}$, respectively, in term singleton pregnancies, and pathologic fetal acidemia with an umbilical artery $\mathrm{pH}<7.0$ occurred in only $0.3 \%$ of cases. In our study population, the NICU admission rate was $6 \%$. These results were quite similar to a previous study from Canada including more than 20,000 singleton infants born at term, in which the mean umbilical artery $\mathrm{pH}$ and $\mathrm{BE}$ were $7.24 \pm 0.07$ and $-5.6 \pm 3.0 \mathrm{mmol} / \mathrm{L}$, respectively, and pathologic fetal acidemia $(\mathrm{pH}<7.0)$ occurred in $0.4 \%$ of cases with a NICU admission rate of $7.6 \%$ [18].

Multiple risk factors, both antepartum and intrapartum, have been reported to be associated with fetal acidemia. Antepartum risk factors include prior caesarean delivery, maternal age 35 years or older, prior neonatal death, single civil status, and short stature [19]. Intrapartum risk factors include the use of oxytocin, tachysystole, meperidine use, uterine rupture, cord complications, clinical indication of chorioamnionitis, meconium-stained amniotic fluid, breech presentation, operative delivery, and abnormal FHR during labor $[10,19-24]$. Our data also confirmed the antepartum factors of lower maternal height and weight, primiparity, lower neonatal birth weight, and SGA as risk factors for low cord pH.

Many studies have investigated the association between meconium staining and possible fetal acidemia, with conflicting results $[25,26]$. One study reported a poor correlation between meconium-stained amniotic fluid and acute acidemia [27]. According to another study that included 323 term pregnancies with meconium-stained amniotic fluid, there was no correlation between umbilical cord acid-base measurements and fetal condition at birth [28]. In contrast, other studies have demonstrated that a severe grade of meconium staining is associated with cord blood $\mathrm{pH}[3,29]$. Our study also confirmed that a high meconium staining grade was associated with lower umbilical cord $\mathrm{pH}$. However, the actual frequency of low cord $\mathrm{pH}(<7.1)$ was low. In detail, the rate of low cord $\mathrm{pH}(<7.1)$ occurred in only $2.87 \%$ of cases of meconium staining grade +1 and $16.67 \%$ of cases of grade +4 . Nuchal cord events increase with gestational age and are found in nearly $25 \%$ of deliveries at term [30]. While a nuchal cord is not associated with adverse outcomes in general, a multiple nuchal cord entanglement may be associated with a risk of meconium, abnormal FHR pattern, the need for operative vaginal delivery, mild umbilical artery acidemia at birth, and lower Apgar scores [31,32]. Our data also identified an incidence of nuchal cord about $30 \%$ in term singleton pregnancies and showed that a multiple nuchal cord entanglement was associated with low cord $\mathrm{pH}$, as expected. The mode of delivery is a well-known factor 


\title{
Obstetrics \& Gynecology Science
}

\author{
Vol. 63, No. 3, 2020
}

associated with low cord $\mathrm{pH}$. Our data showed that the rate of low cord pH was higher in vacuum-assisted delivery than in any other mode of delivery, reflecting clinical situations requiring prompt delivery in cases of fetal jeopardy. However, the actual incidence of low cord pH ( $<7.1)$ was only $2.84 \%$, while that of significant acidemia $(<7.0)$ was exceedingly low $(0.65 \%)$ in cases with vacuum-assisted delivery.

A population-based study indicated that the incidence of NE was 3.0 per 1,000 live births (95\% confidence interval $[\mathrm{Cl}], 2.7-3.3)$ and that of HIE was 1.5 per 1,000 live births (95\% Cl, 1.3-1.7) [33]. According to our study, the incidence of significant acidemia $(<7.0)$ was $0.3 \%$ and that of umbilical cord $\mathrm{pH}<7.1$ was $1.1 \%$. Our observation that $1.8 \%$ of cases with low cord $\mathrm{pH}(<7.1)$ were finally presumed to have CP was similar to that of a recent observational cohort study including 51,519 term singleton neonates in which only $2.96 \%$ of neonates with cord $\mathrm{pH}<7.0$ had adverse neurologic outcomes, including encephalopathy with seizures and/ or death [34]. In their study, the authors concluded that most neonates with neurological morbidity have normal cord $\mathrm{pH}$ values, which is consistent with our data showing that most infants with neurodevelopmental disability during the same study period had normal umbilical cord pH levels. Collectively, these population-based studies suggest that variables other than acidemia are influencing the neurologic outcomes of infants.

Our study showed that an ultrasound of the brain was performed in only about one-third (28.8\%) of cases with low cord $\mathrm{pH}$, among which an abnormal finding was observed in $27.7 \%$. In fact, diverse findings were identified, including diffuse brain edema, grade 2 intraventricular hemorrhage, basal ganglia vasculopathy, Dandy-Walker complex, and myelination disorder, which reflects the various underlying pathologies among cases of low cord pH. According to an MRI study of children with CP from a cohort of 334,339 infants at $\geq 36$ weeks of gestation, it was demonstrated that only $5 \%$ had imaging abnormalities compatible with hypoxic-ischemic brain injury [35]. Other abnormalities included focal arterial infarction (22\%), brain malformation (14\%), periventricular white matter abnormalities (12\%), generalized brain atrophy $(7 \%)$, intracranial hemorrhage $(5 \%)$, and delayed myelination $(2 \%)$. Of note, a normal brain was seen in almost onethird of infants who underwent neuroimaging (31\%).

The limitations of our study can be summarized as follows. First, considering the characteristics of a tertiary center where term neonates without NICU admission may not have longterm follow-up, we were unable to obtain complete information on the long-term outcomes of all neonates in this hospital population, especially those with cord $\mathrm{pH}>7.1$, which may have resulted in an underestimation of the total prevalence of CP. Importantly, to overcome this limitation, we tried to search for CP cases among all the infants delivered in our hospital based on ICD-10-CM codes in our electronic medical record system, and we found 18 cases during the same study period that were diagnosed (or presumed) with CP later in life. The average follow-up period for 181 cases (163 cases with low cord $\mathrm{pH}$ and 18 cases of $\mathrm{CP}$ with normal cord $\mathrm{pH}$ ) was 3.7 years. As a result, there were $21 \mathrm{CP}$ cases, including 3 cases with low cord pH (2 cases of HIE-related and 1 case of ataxic (P), among 14,221 term singleton deliveries. Collectively, our data indicate that the total prevalence of CP in term pregnancies can be estimated at 1.48 per 1,000 live births, of which HIE-associated CP accounts for $9.5 \%$ (2 out of 21). This proportion seems to be similar to our previous study with a different study period (from October 1994 to December 2004) in which perinatal HIE events were evident in $20 \%$ of term CP cases [36]. Second, given the relatively higher proportion of high-risk pregnancies in our population, as reflected by the $6 \%$ NICU admission rate in term singleton pregnancies, generalizability to low risk population may be also limited. Thirdly, although vanishing twin was one of the criteria for exclusion from our study population, there is a chance that not all vanishing twin cases were ruled out in our population since clinical information in the first trimester may be not completely collected.

One of the strengths of our study is that we included a large number of consecutive deliveries in which the performance rate of cord ABGA was over $90 \%$, thereby precluding intrinsic selection bias from case-control studies. In addition, we presented the objective association between umbilical cord $\mathrm{pH}$ and certain intrapartum factors including meconium staining, which is often overestimated in medicolegal disputes. Our data showing a very low incidence of HIEassociated CP even in neonates with low cord pH $(1.23 \%, 2$ out of 163) and a relatively high proportion of normal cord $\mathrm{pH}$ among total CP cases strongly support the expert opinion that if comprehensive etiologic evaluation is not possible, the term HIE should be replaced by NE, since neither hypoxia nor ischemia can be assumed to have been the unique causal mechanism [14]. 


\section{Obstetrics \& Gynecology Science}

Ji Hee Lee, et al. Cord arterial blood gas analysis in term pregnancies

\section{Conflict of interest}

No potential conflict of interest relevant to this article was reported.

\section{Ethical approval}

The retrospective study was approved by the Institutional Review Board of Samsung Medical Center (IRB file No. 201908-028-002) and performed in accordance with the principles of the Declaration of Helsinki.

\section{References}

1. Gary CF, Bloom SL, Dashe JS, Spong CY, Hoffman BL, Casey BM, et al. Williams obstetrics. 25th ed. New York (NY): McGraw-Hill Medical; 2018.

2. Manley BJ, Owen LS, Hooper SB, Jacobs SE, Cheong JL, Doyle LW, et al. Towards evidence-based resuscitation of the newborn infant. Lancet 2017;389:1639-48.

3. Barber CA, Wyckoff MH. Use and efficacy of endotracheal versus intravenous epinephrine during neonatal cardiopulmonary resuscitation in the delivery room. Pediatrics 2006;118:1028-34.

4. Weiner GM. Textbook of neonatal resuscitation. 7th ed. Chicago (IL): American Academy of Pediatrics and American Heart Association; 2016.

5. Blume HK, Loch CM, Li Cl. Neonatal encephalopathy and socioeconomic status: population-based case-control study. Arch Pediatr Adolesc Med 2007;161:663-8.

6. Blume HK, Li Cl, Loch CM, Koepsell TD. Intrapartum fever and chorioamnionitis as risks for encephalopathy in term newborns: a case-control study. Dev Med Child Neurol 2008;50:19-24.

7. Cowan F, Rutherford M, Groenendaal F, Eken P, Mercuri $\mathrm{E}$, Bydder GM, et al. Origin and timing of brain lesions in term infants with neonatal encephalopathy. Lancet 2003;361:736-42.

8. Badawi N, Kurinczuk JJ, Keogh JM, Alessandri LM, O'Sullivan F, Burton PR, et al. Intrapartum risk factors for newborn encephalopathy: the Western Australian casecontrol study. BMJ 1998;317:1554-8.

9. Gillam-Krakauer M, Carter BS. Neonatal hypoxia and seizures. Pediatr Rev 2012;33:387-96.

10. Rodríguez Fernández V, López Ramón Y Cajal CN, Marín Ortiz E, Couceiro Naveira E. Intrapartum and perinatal results associated with different degrees of staining of meconium stained amniotic fluid. Eur J Obstet Gynecol Reprod Biol 2018;228:65-70.

11. Blickstein I, Green T. Umbilical cord blood gases. Clin Perinatol 2007;34:451-9.

12. ACOG Committee on Obstetric Practice. ACOG Committee Opinion No. 348, November 2006: Umbilical cord blood gas and acid-base analysis. Obstet Gynecol 2006;108:1319-22.

13. National Collaborating Centre for Women's and Children's Health. Intrapartum care: care of healthy women and their babies during childbirth. London: National Institute for Health and Care Excellence; 2007.

14. Executive summary: Neonatal encephalopathy and neurologic outcome, second edition. Report of the American College of Obstetricians and Gynecologists' Task Force on Neonatal Encephalopathy. Obstet Gynecol 2014;123:896-901.

15. Sameshima H, Ikenoue T, Ikeda T, Kamitomo M, Ibara S. Unselected low-risk pregnancies and the effect of continuous intrapartum fetal heart rate monitoring on umbilical blood gases and cerebral palsy. Am J Obstet Gynecol 2004;190:118-23.

16. Sameshima H, Ikenoue T. Predictive value of late decelerations for fetal acidemia in unselective low-risk pregnancies. Am J Perinatol 2005;22:19-23.

17. Cahill AG, Roehl KA, Odibo AO, Macones GA. Association and prediction of neonatal acidemia. Am J Obstet Gynecol 2012;207:206.e1-206.e8.

18. Victory R, Penava D, Da Silva O, Natale R, Richardson B. Umbilical cord $\mathrm{pH}$ and base excess values in relation to adverse outcome events for infants delivering at term. Am J Obstet Gynecol 2004;191:2021-8.

19. Maisonneuve E, Audibert F, Guilbaud L, Lathelize J, Jousse $M$, Pierre $F$, et al. Risk factors for severe neonatal acidosis. Obstet Gynecol 2011;118:818-23.

20. Wretler S, Nordström L, Graner S, Holzmann M. Risk factors for intrapartum acidemia - a cohort study. J Matern Fetal Neonatal Med 2018;31:3232-7.

21. Cahill $A G$, Spain J. Intrapartum fetal monitoring. Clin Obstet Gynecol 2015;58:263-8.

22. Milsom I, Ladfors L, Thiringer K, Niklasson A, Odeback A, 


\section{Obstetrics \& Gynecology Science}

Vol. 63, No. 3, 2020

Thornberg E. Influence of maternal, obstetric and fetal risk factors on the prevalence of birth asphyxia at term in a Swedish urban population. Acta Obstet Gynecol Scand 2002;81:909-17.

23. Herbst $A$, Wolner-Hanssen $P$, Ingemarsson I. Risk factors for acidemia at birth. Obstet Gynecol 1997;90:125-30.

24. Jonsson M, Nordén-Lindeberg S, Ostlund I, Hanson U. Acidemia at birth, related to obstetric characteristics and to oxytocin use, during the last two hours of labor. Acta Obstet Gynecol Scand 2008;87:745-50.

25. Nelson KB, Ellenberg JH. Obstetric complications as risk factors for cerebral palsy or seizure disorders. JAMA 1984;251:1843-8.

26. Moster D, Wilcox AJ, Vollset SE, Markestad T, Lie RT. Cerebral palsy among term and postterm births. JAMA 2010;304:976-82.

27. Hutton EK, Thorpe J. Consequences of meconium stained amniotic fluid: what does the evidence tell us? Early Hum Dev 2014;90:333-9.

28. Yeomans ER, Gilstrap LC 3rd, Leveno KJ, Burris JS. Meconium in the amniotic fluid and fetal acid-base status. Obstet Gynecol 1989;73:175-8.

29. Perveen F, Khan A, Ali T, Rabia S. Umbilical cord blood $\mathrm{pH}$ in intrapartum hypoxia. J Coll Physicians Surg Pak
2015;25:667-70.

30. Larson JD, Rayburn WF, Harlan VL. Nuchal cord entanglements and gestational age. Am J Perinatol 1997; 14:555-7.

31. Larson JD, Rayburn WF, Crosby S, Thurnau GR. Multiple nuchal cord entanglements and intrapartum complications. Am J Obstet Gynecol 1995;173:1228-31.

32. Onderoğlu LS, Dursun P, Durukan T. Perinatal features and umbilical cord blood gases in newborns complicated with nuchal cord. Turk J Pediatr 2008;50:466-70.

33. Kurinczuk JJ, White-Koning M, Badawi N. Epidemiology of neonatal encephalopathy and hypoxic-ischaemic encephalopathy. Early Hum Dev 2010;86:329-38.

34. Yeh P, Emary K, Impey L. The relationship between umbilical cord arterial $\mathrm{pH}$ and serious adverse neonatal outcome: analysis of 51,519 consecutive validated samples. BJOG 2012;119:824-31.

35. Wu YW, Croen LA, Shah SJ, Newman TB, Najjar DV. Cerebral palsy in a term population: risk factors and neuroimaging findings. Pediatrics 2006;118:690-7.

36. Koo YA, Choi DS, Choi SJ, Oh SY, Chang YS, Park WS, et al. Clinical characteristics of cerebral palsy following preterm or term birth; 10 years' experience. Korean J Obstet Gynecol 2006;49:2544-9. 